\title{
Expression of E-selectin ligand-1 (CFR/ESL-1) on hepatic stellate cells: Implications for leukocyte extravasation and liver metastasis
}

\author{
MARIANNE ANTOINE ${ }^{1}$, CARMEN G. TAG ${ }^{2}$, AXEL M. GRESSNER ${ }^{2}$, \\ CLAUS HELLERBRAND $^{3}$ and PAUL KIEFER ${ }^{1}$
}

${ }^{1}$ Institute of Clinical Chemistry and Laboratory Medicine, University of Regensburg; ${ }^{2}$ Institute of Clinical Chemistry and
Pathobiochemistry, RWTH Aachen; ${ }^{3}$ Department of Internal Medicine I, University of Regensburg, Germany

Received October 22, 2007; Accepted February 5, 2008

DOI: $10.3892 /$ or_00000230

\begin{abstract}
Leukocytes and tumor cells use E-selectin binding ligands to attach to activated endothelial cells expressing Eselectin during inflammation or metastasis. The cysteine-rich fibroblast growth factor receptor (CFR) represents the main E-selectin ligand (ESL-1) on granulocytes and its expression is exclusively modified by $\alpha(1,3)$-fucosyltransferases IV or VII (FucT4 and FucT7). Hepatic stellate cells (HSC) are pericytes of liver sinusoidal endothelial cells. The activation of HSC and transdifferentiation into a myofibroblastic phenotype is involved in the repair of liver tissue injury, liver regeneration and angiogenesis of liver metastases. In the present study, we demonstrated that HSC expressed CFR together with FucT7 and exhibited a functional E-selectin binding activity on their cell surface. Since HSC appear to be oxygen-sensing cells, the expression of E-selectin binding activity was analyzed in HSC under a hypoxic atmosphere. While the expression of the glycoprotein CFR was unaffected by hypoxia, the cellassociated E-selectin binding activity decreased. However, under the same conditions, mRNA expression of the modifying enzyme FucT7 increased. The loss of E-selectin binding activity, therefore, appears to be neither the result of a reduced expression of the modifying transferase nor the expression of the backbone glycoprotein. After the transient transfection of HSC with CFR cDNA, the E-selectin binding activity (ESL-1) was efficiently released into the supernatant. Therefore, we hypothesize that under hypoxia, ESL-1 is shed from activated HSC. Our findings provide a novel perspective on the function of HSC in liver metastasis and inflammatory liver diseases.
\end{abstract}

Correspondence to: Dr Claus Hellerbrand, Department of Internal Medicine I, University of Regensburg, D-93042 Regensburg, Germany

E-mail: claus.hellerbrand@klinik.uni-regensburg.de

Abbreviations: CFR, cysteine-rich fibroblast growth factor receptor; HSC, hepatic stellate cells; ESL-1, E-selectin ligand-1

Key words: E-selectin, E-selectin ligand-1, hepatic stellate cells

\section{Introduction}

Hepatic stellate cells (HSC) are pericytes of liver sinusoidal endothelial cells. HSC contact hepatocytes and endothelial cells via long cytoplasmic processes, which may be important for cell-cell communication and the structural (re)organization of the hepatic sinusoidal compartment. The activation of HSC and transdifferentiation into a myofibroblastic phenotype (MFB, myofibroblasts) appear to be involved in the repair of liver tissue injury, regeneration and angiogenesis of liver metastases $(1,2)$.

The entry of leukocytes as well as tumor cells is controlled by cell adhesion particularly between the invading and endothelial cells. The cytokine-inducible E-selectin mediates the binding of neutrophils to endothelial cells and is implicated in colon cancer metastasis (3).

E-selectin is a calcium-dependent type-I transmembrane glycoprotein with an extracellular lectin-like domain that interacts with sialylated carbohydrates. E-selectin is synthesized by activated endothelial cells and mediates the initial tethering of circulating blood cells with endothelial cells. Its expression is transcriptionally up-regulated by several factors including tumor necrosis factor (TNF), interleukin (IL)-1, nuclear factor $\mathrm{\kappa B}(\mathrm{NF}-\mathrm{\kappa B})$ and under hypoxia $(4,5)$.

Binding to E-selectin depends on the presence of sialylated and fucosylated carbohydrate structures (sialyl Lewis Xlike) which are generated by $\alpha(1,3)$-fucosyltransferases (FucT) (6). E-selectin ligand-1 (ESL-1), P-selectin glycoprotein ligand-1 (PSGL-1) and CD44 represent E-selectin ligand activities on neutrophils. While PSGL-1 plays a major role in the initial leukocyte capture, ESL-1 is critical for converting initial tethers into steady slow rolling (7).

ESL-1 is the major E-selectin ligand on neutrophils and solely binds to E-selectin. Its expression is almost exclusively modified by FucT4 and FucT7. ESL-1 is identical to the cysteine-rich fibroblast growth factor receptor (CFR) and the membrane sialoglycoprotein of the medial cisternae of the Golgi complex, MG-160. The gene-encoding ESL-1/CFR is known as GLG1 (Golgi complex-localized glycoprotein-1) (8).

E-selectin ligands mediate adhesive interactions between hematopoietic progenitor and bone marrow endothelial cells. It can also be assumed that tumor cells use E-selectin ligand 

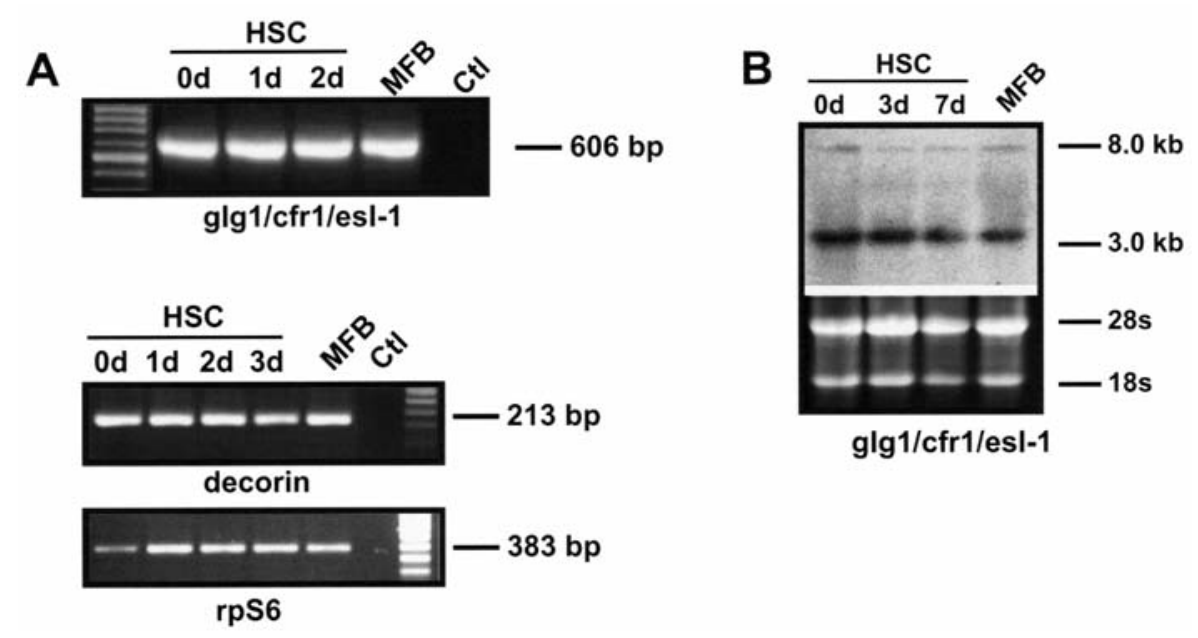

Figure 1. The E-selectin ligand-1/CFR encoding gene GLG1 is equally expressed in HSC during in vitro transdifferentiation. The transcription of GLG1 (CFR1, ESL-1) was analyzed by (A) RT-PCR and (B) Northern blot analysis in i) freshly isolated HSC (day 0), ii) HSC cultured for 1-3 days and iii) secondary cultures of primary HSC fully transdifferentiated into a myofibroblastic phenotype (MFB). Decorin and the ribosomal protein S6 (rpS6) mRNA served as loading controls.

to adhere to endothelial and stromal cells after extravasation (9). In an experimental hepatic metastasis model, HSC, the only mesenchymal cells present in the extravascular space of the sinusoidal compartment, demonstrated a close interaction with colon carcinoma cells (10). The adhesion molecules involved in this interaction are unknown thus far but may be potential targets for new therapeutic strategies.

In this study, we analyzed the expression of CFR/ESL-1, fucosyltransferases and E-selectin in primary HSC and in in vitro transdifferentiated/activated HSC. We investigated their gene expression under hypoxia which represents the microenvironmental atmosphere present in inflamed tissue and in tissue infiltrated by rapidly growing tumor cells.

To the best of our knowledge, the expression of E-selectin has thus far been exclusively reported on endothelial cells. Herein, we reported the expression of E-selectin and its ligand ESL-1 by HSC. Notably, under hypoxia, FucT7 was up-regulated and accompanied by a strong induction of the E-selectin expression.

\section{Materials and methods}

Preparation of cells. Rat HSC were prepared from male Sprague-Dawley rats by the pronase/collagenase method as previously described (11). The mean purity was $>95 \%$ and the yield ranged from 25 to $50 \times 10^{6}$ cells/liver. Activated rat HSC/myofibroblasts (MFB) were generated by secondary cultures of trypsinized 7-day-old primary HSC. Rat HSC and MFB were cultured in DMEM supplemented with $10 \%$ FCS (11).

The human HSC line LX-2 (which was a kind gift from Dr S.L. Friedman, Mount Sinai Medical School, New York, NY) was kept at $2 \%$ FCS. Cells were routinely cultured in humidified $95 \%$ air $\left(20 \% \mathrm{O}_{2}\right)$ and $5 \% \mathrm{CO}_{2}$ at $37^{\circ} \mathrm{C}$. For the hypoxic stimulation, cells were serum-starved $(0.5 \%$ FCS $)$ for $24 \mathrm{~h}$ and subsequently placed in an Invivo 2 hypoxia incubator (Invivo2 hypoxia workstation 400, Ruskinn Technology Guiseley Leeds, UK) and flushed with gas $\left(0.5 \% \mathrm{O}_{2}, 5 \% \mathrm{CO}_{2}\right.$ and $\left.94.5 \% \mathrm{~N}_{2}\right)$ for $48 \mathrm{~h}$.
RNA isolation and RT-PCR analysis. Total RNA from LX-2 cells was extracted by using the RNeasy mini kit (Qiagen, Hilden, Germany). RNA from primary rat HSC was extracted with the RNeasy lipid tissue mini kit (Qiagen) according to the manufacturer's instructions. Of the total extracted RNA, $1 \mu \mathrm{g}$ was reverse transcribed with an omniscript reverse transcriptase kit (Qiagen) using oligo dT as a primer. cDNAs were amplified using the Taq DNA polymerase kit from Qiagen. PCR primers were obtained from Invitrogen (Heidelberg, Germany). The primer pairs for PCR-analysis were: 5'-GTC CAG TTG TAA GTT CTC CTC TGA-3' (E-sel forward), 5'-GCA AGC AAA ATA TAA ATC CCG GTC-3' (E-sel reverse), 5'-GAA GAG TCC TGC AGG GAG GAC-3' (GLG1 forward), 5'-GCA AGC AAA ATA TAA ATG CCG GTC-3' (GLG1 reverse), 5'-TCA CCA TCC TTG TCT GGC ACTG-3' (FucT7 forward), 5'-GCT CAG CAC CCA GTT GAA GAT G-3' (FucT7 reverse), 5'-CG CTG GGT TTG GAT GAA CTT CGA-3' (FucT4 forward), 5'-ACT TGG GAA GTC GTC CAC GTG GAT G A-3' (FucT4 reverse), 5'-CTC TGG CAT AAT CCT TAC GAC-3' (decorin forward) and 5'-GGT ATG CAA GTC CTT CAG GTT C-3' (decorin reverse). The rat primers used for the amplification of VEGF and rpS6 have been published before (11). To confirm the specificity of the amplification, PCR products were DNA sequenced using the big dye terminator v1.1 cycle sequencing kit and an ABI PRISM 310 genetic analyzer (Applied Biosystems, Weiterstadt, Germany).

Northern blot analysis. Total RNA isolated from monolayer cultures was separated using $1 \%$ formaldehyde agarose gels and blotted onto a nylon membrane (Hybond $\mathrm{N}^{+}$, Amersham Pharmacia Biotech, Buckinghamshire, UK). cDNA probes for hybridization were prepared by labeling a $606 \mathrm{bp}$ purified PCR fragment of the rat CFR cDNA with [ $\left.{ }^{32} \mathrm{P}\right] \mathrm{dCTP}$ using a random primer labeling system (Invitrogen).

Immunofluorescence. Three-day old rat HSC and MFB were cultured on glass coverslips coated with polylysine or collagen at a density of $2 \times 10^{4}$ cells/35-mm dish. For immunostaining, 

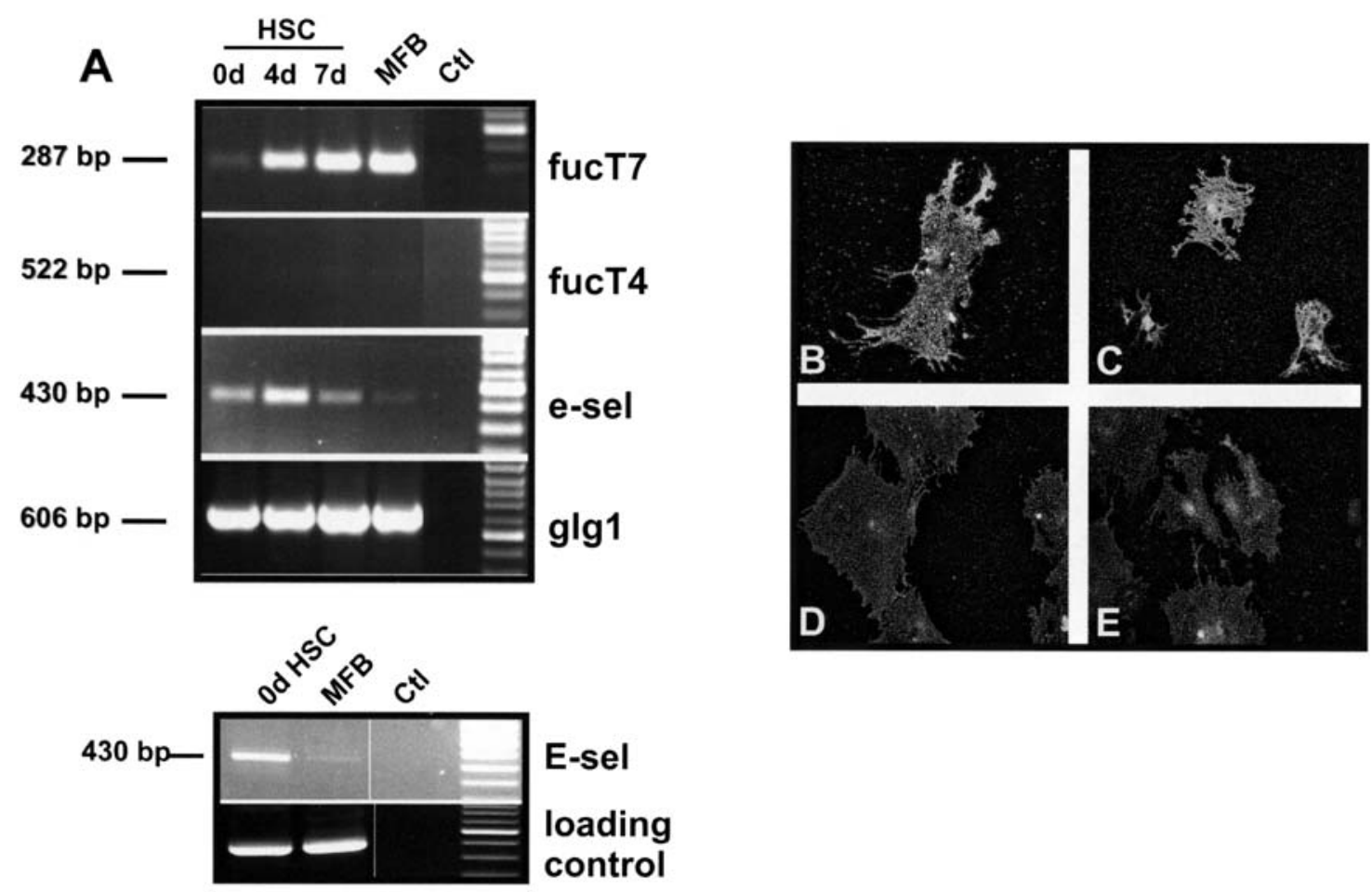

Figure 2. Expression of $\alpha(1,3)$-fucosyltransferases, E-selectin and E-selectin ligand activity by HSC. (A) Transcription of $\alpha(1,3)$-fucosyltransferases 4 and 7 (FucT4 and FucT7) and E-selectin (E-sel) was analyzed by RT-PCR in i) freshly isolated HSC (day 0), ii) HSC cultured for 4 or 7 days and iii) secondary cultures of primary HSC fully transdifferentiated into a myofibroblastic phenotype (MFB). The GLG1 transcription served as a loading control (upper panel). An additional semiquantitative RT-PCR analysis with limited cycles was performed to compare E-selectin mRNA expression in freshly isolated HSC (d0) and MFB (lower panel). (B-E) E-selectin ligand activity was analyzed by immunofluorescence by applying a mouse E-selectin human Ig chimera and HSC cultured for 2 days (B and C) and MFB (D and E) cultured on collagen (B and D) or polylysine, respectively (C and E). Cells were fixed with paraformaldehyde but not permeabilized.

cells were fixed with $4 \%(\mathrm{w} / \mathrm{v})$ paraformaldehyde in PBS. Non-specific binding sites were blocked with $3 \%(\mathrm{v} / \mathrm{v})$ bovine serum albumin (BSA) in PBS. Cells were incubated with a recombinant mouse E-selectin/human immunoglobulin chimera (R\&D Systems, Wiesbaden, Germany). The immunocomplexes were detected using an appropriated fluorescence dye-conjugated secondary antibody. The cells were mounted in $90 \%$ glycerol containing p-phenylenediamine and viewed with a $63 \mathrm{x}$ oil immersion lens on a Zeiss microscope equipped with barrier filters for fluorescein.

Immunoblot analysis. The procedures used for preparing cell lysates have been described in detail elsewhere (11). Samples from equivalent numbers of cells were fractionated by SDSPAGE in $12.5 \%$ gels, transferred to nitrocellulose membranes (Schleicher \& Schuell), probed with a recombinant mouse Eselectin/human immunoglobulin chimera or with a rabbit polyclonal antiserum kindly provided by C.J. Dimitroff (Harvard Institute of Medicine, Boston, MA). Subsequently, horseradish peroxidase-linked secondary antibodies (Pierce) were added and immunocomplexes were detected by enhanced chemiluminescence (Pierce).

Statistical analysis. Statistical analyses were performed using SPSS version 10.0 (SPSS, Chicago, IL, USA). Results are expressed as mean \pm SD (range). A comparison between the groups was made using the Student's unpaired t-test. P-values $<0.01$ and $<0.05$ were considered significant.

\section{Results}

The E-selectin ligand-1/CFR-encoding gene GLG1 is expressed in freshly isolated and activated HSC. Expression of the E-selectin ligand-1/CFR-encoding gene GLG1/CFR was analyzed in hepatic stellate cells (HSC) at different time points during in vitro activation/transdifferentiation to myofibroblasts (MFB) by RT-PCR (Fig. 1A). The identity of the PCR fragments was confirmed by DNA sequencing. GLG1 was clearly detectable in all cells analyzed and did not appear to be differentially expressed during the in vitro activation process of HSC.

GLG1 mRNA expression was further examined by Northern blot analysis, revealing two transcripts of the expected size of $\sim 3$ and $8 \mathrm{~kb}$, respectively (Fig. 1B). GLG1 gene expression levels also appeared invariant in HSC due to Northern blot analysis during the course of transdifferentiation into myofibroblasts.

HSC and MFB express the $\alpha(1,3)$-fucosyltransferase VII. The E-selectin ligand activity correlates with the selective generation of the sialyl Lewis $x$-like epitope and the HECA452-reactive carbohydrate epitope, which depends on the expression of the $\alpha(1,3)$-fucosyltransferase IV (FucT4) or VII (FucT7). In cells expressing FucT4 or FucT7, CFR/ESL-1 is the preferred target protein for the generation of E-selectinbinding carbohydrate modifications (6). Therefore, RNA expression of candidate fucosyltransferases was analyzed by 

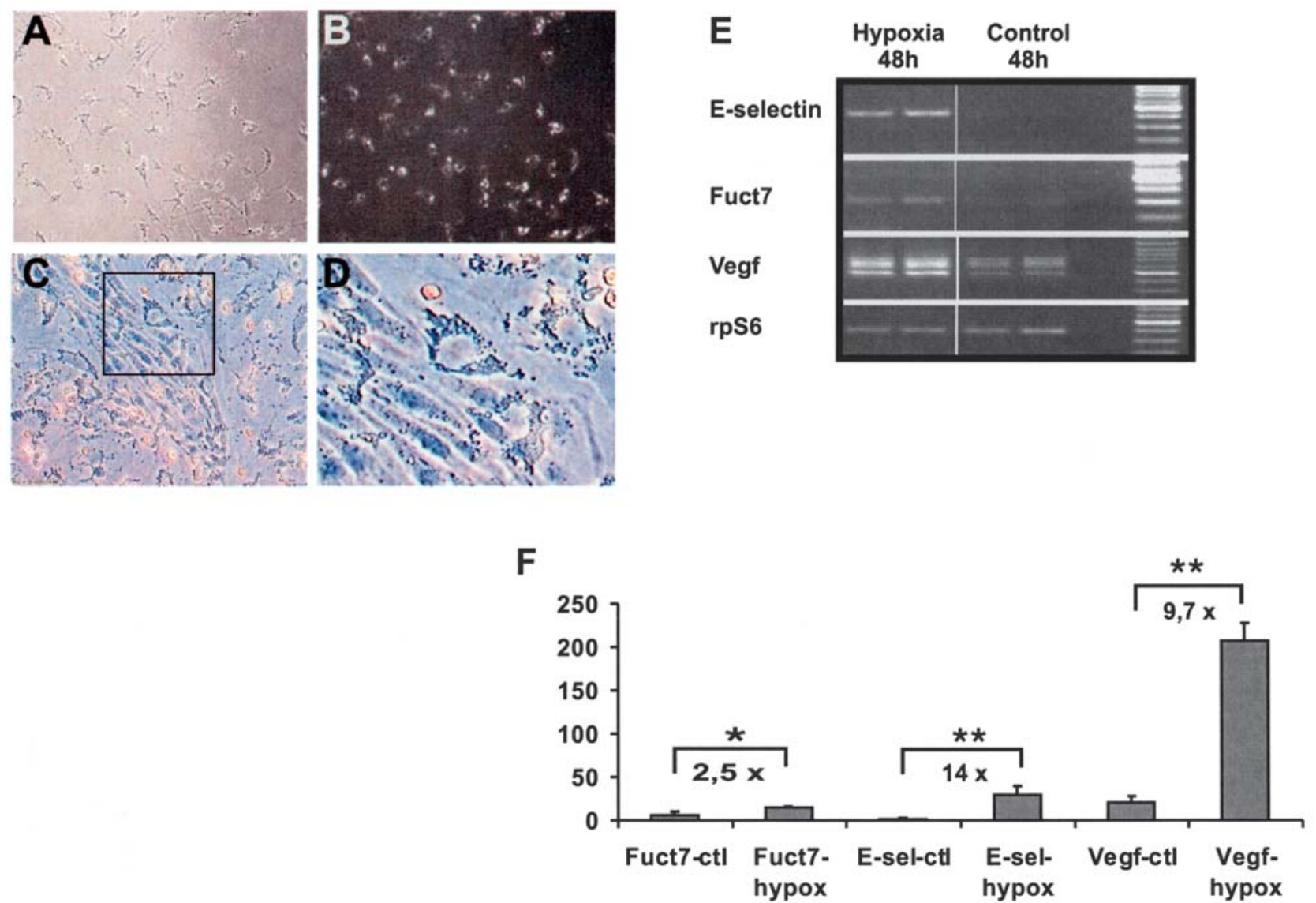

Figure 3. The up-regulation of E-selectin and $\alpha(1,3)$-fucosyltransferase VII in HSC by hypoxia. HSC were cultured for 2 days under normal atmospheric oxygen or hypoxic conditions $\left(0.5 \% \mathrm{O}_{2}, 5 \% \mathrm{CO}_{2}\right.$ and $\left.94.5 \% \mathrm{~N}_{2}\right)$. Under normal conditions HSC showed a typical morphology (A) and characteristic vitamin-A associated autofluorescence (B). Under the hypoxic atmosphere, elongated cells appeared, which had lost their lipid droplets and exhibited a fibroblastic phenotype (C and D, enlarged section of C). (E and F) E-selectin, fucosyltransferase 7 (FucT7) and VEGF were analyzed by RT-PCR analysis. Fig. 3E shows the RT-PCR amplification products of two independent experiments of HSC cultured for $48 \mathrm{~h}$ under hypoxic conditions (hypoxia) or normal atmospheric oxygen conditions (control). RT-PCR amplification products were quantified by densitometry and normalized to rpS6 mRNA as an internal control. Image analysis was performed with Kodak 1-D image analysis software. (F) Values presented are the means \pm SD of three independent experiments. Differences between experimental groups were analyzed by the unpaired Student's t-test. ${ }^{* *} \mathrm{P}<0.01$ and ${ }^{*} \mathrm{P}<0.05$ were considered significant.

RT-PCR in HSC at different time points during the in vitro activation. An increased mRNA expression of FucT7 was found during the in vitro transdifferentiation of HSC. The amplified PCR fragments have been confirmed by DNA sequencing. In contrast to the human HSC cell line, LX-2, which expressed FucT4 and FucT7 transcripts, FucT4 mRNA was not detected in rat HSC regardless of the activation stage (Fig. 2A).

The E-selectin encoding gene is expressed in HSC and downregulated in $M F B$. Since HSC interact with hepatocytes and endothelial cells during the early phases of liver regeneration, and with cancer cells and leukocytes under pathological conditions including metastasis and inflammation, cells known to express E-selectin-binding sites, we investigated the RNA expression of E-selectin by HSC and MFB. Notably, RT-PCR showed that HSC and MFB expressed E-selectin transcripts.

A semiquantitative RT-PCR analysis with limited cycles demonstrated a clear down-regulation of E-selectin mRNA in MFB compared to the freshly isolated HSC (Fig. 2A, lower panel). The amplification of the GLG1/CFR transcript was used as an internal control. The amplified PCR fragments have been confirmed by DNA sequencing.
E-selectin ligand is expressed on HSC and MFB. To investigate the surface expression of the functional E-selectin binding activity on HSC during the course of in vitro activation, immunofluorescence was performed with cells grown on cover slips coated with collagen or polylysine. Cells were fixed and incubated with a mouse E-selectin human Ig chimera. Although during in vitro transdifferentiation the transcription of GLG1 seems to be invariant and FucT7 is increased, respectively, activated $\mathrm{HSC} / \mathrm{MFB}$ appeared to have less E-selectin ligands present on their cell surface than did HSC three days after isolation, and this appears to be independent of the cell culture substrate (Fig. 2B-D).

The up-regulation of E-selectin and $\alpha(1,3)$-fucosyltransferase VII in HSC by hypoxia. During liver repair processes, the inflammatory affection of the sinusoidal compartment and of liver metastasis HSC have to cope with a hypoxic environment and are considered as hypoxia-sensitising cells (12). Therefore, we cultured HSC under normal atmospheric oxygen and hypoxic conditions to study gene expression. After 3 days in the culture under normal atmospheric oxygen conditions HSC exhibited the typical stellate-like cell morphology with lipid droplets (Fig. 3A) and all cells showed the typical auto- 


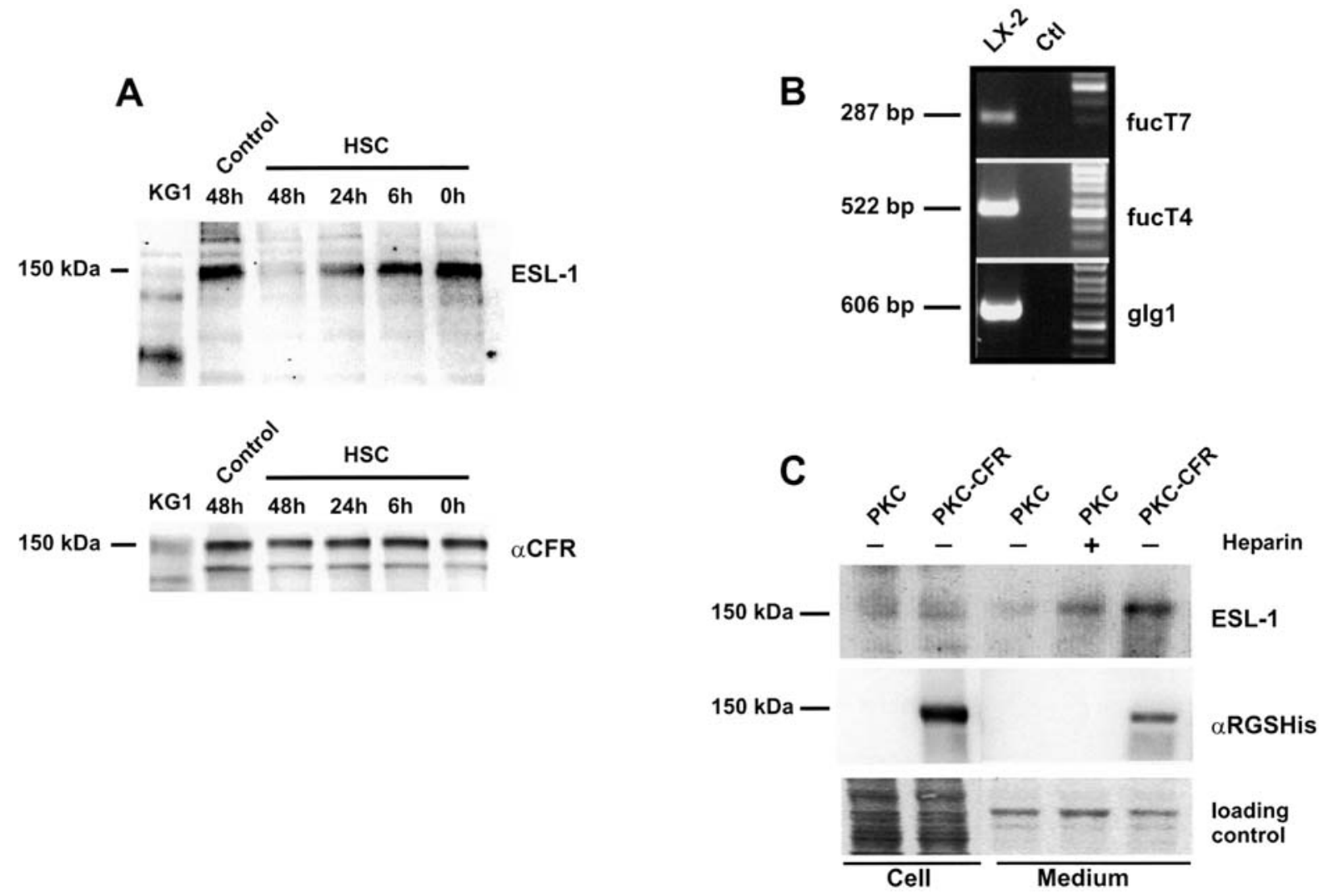

Figure 4. Cell-associated ESL-1 is down-regulated on HSC under hypoxia. (A) HSC were cultured for 2 days under normal atmospheric oxygen (control) or hypoxic conditions for different time intervals $(0-48 \mathrm{~h})$. Western blotting was performed by applying a mouse E-selectin-FC chimera to examine E-selectin binding (upper panel), or a polyclonal antibody against the protein backbone to investigate the expression of total ESL-1/CFR protein (lower panel). (B) The transcription of $\alpha(1,3)$-fucosyltransferases 4 and 7 (FucT4 and FucT7) and of GLG1 was analyzed by RT-PCR in LX-2 cells. (C) Analysis of E-selectin binding activity in the cell extracts and supernatant from LX-2 cells by immunoblotting with E-selectin-immunoglobulin (upper panel) and anti-His antibody (middle panel). For some conditions LX-2 cells were transfected with cDNA encoding the highly conserved His-tagged chicken CFR. Since CFR is a heparinbinding protein, in one experiment, heparin was added to the medium. Protein loading was controlled by Ponceau S red staining (lower panel).

fluorescence-associated vitamin-A storage (Fig. 3B). Under the hypoxic atmosphere a significant part of the cells showed an elongated, fibroblast-like cell morphology (Fig. 3C and D, enlarged section). Some of these activated HSC had already lost their lipid droplets (Fig. 3D).

We then investigated gene expression in cultured HSC under normal atmospheric oxygen and hypoxic conditions using RT-PCR techniques.

The RT-PCR analysis showed a marked increase in the gene expression of E-selectin (14-fold) and Fuct7 (2.5-fold) in hypoxic HSC. Accordingly, the known hypoxia-inducible gene VEGF revealed a significant induction (9.7-fold) (Fig. 3F).

Cell-associated ESL-1 is down-regulated on HSC under hypoxia. To examine the influence of hypoxia on the expression of E-selectin binding activities and CFR/ESL-1, we immunoblotted extracts prepared from HSC cultured under normal atmospheric oxygen and hypoxic conditions for $48 \mathrm{~h}$, respectively. The membranes were probed with E-selectin human immunoglobulin chimera and with rabbit polyclonal anti-ESL-1 antiserum. Although the protein expression of ESL-1/CFR remained at a constant level (Fig. 4A, lower panel), the cell-associated E-selectin binding activity clearly decreased under hypoxia (Fig. 4A, upper panel). Extracts from the human hematopoietic progenitor cell line KG1a were included as a control. The E-selectin binding activities present on KG1a are represented by CD44 at $100 \mathrm{kDa}$ and PSGL-1 at $130 \mathrm{kDa}$, while ESL-1/CFR expression was not detected (Fig. 4A) (14). In contrast, HSC exclusively expressed a single band of $150 \mathrm{kDa}$ corresponding to the E-selectin binding activity.

Previously, we showed that CFR/ESL-1 can be released into the supernatant by COS-1 cells (13). The LX-2 HSC line represents a well-established model of activated HSC. LX-2 cells express ESL1/CFR and particularly the leukocyte fucosyltransferase IV, which is the main Lewis $\mathrm{x}$ carbohydratemodifying enzyme in granulocytes (Fig. 4B). Therefore, we analyzed the cell extracts and supernatant from the LX-2 cells for E-selectin binding activity by immunoblotting with E-selectin immunoglobulin. Cell-associated E-selectin binding activity was detected in the LX-2 cells, but considerably more activity was found in the supernatant, particularly when heparin was included in the medium (Fig. 4C). To demonstrate that ESL-1 represents the E-selectin binding activity, LX-2 cells were transfected with cDNA encoding the highly conserved chicken CFR which has been His-tagged. In the transfected cells, a slightly more cell-associated E-selectin binding activity was detectable, but considerably more activity was found in the supernatant suggesting that CFR was efficiently modified to ESL-1 in LX-2 cells and was 
secreted or produced by shedding into the supernatant. The same extracts were analyzed with an anti-His antibody to demonstrate the expression of the transfected cDNA.

\section{Discussion}

In the present study, we demonstrated for the first time functional E-selectin binding sites on HSC. Our results suggest that the E-selectin activity expressed by HSC is mainly represented by CFR which is modified by the fucosyltransferase FucT7. A hypoxic atmosphere substantially induced the transcription of the modifying fucosyltransferase FucT7 as well as transcription of E-selectin in HSC. Under hypoxia, ESL-1 appears to be shed from the HSC surface into the supernatant. The soluble form of ESL-1 is still capable of binding to E-selectin, and thus, may compete with cellassociated ESL-1.

Leukocyte recruitment to sites of inflammation involves several types of adhesion molecules and their receptors, the selectins (E-, P- and L-), integrins and members of the immunoglobulin family (14). According to current models, the first contact between leukocytes and endothelial cells is mediated via selectin-ligand interactions which lead to the attachment and rolling of the leukocytes on the activated endothelial cells. In a very similar way selectins may facilitate cancer metastasis and tumor cell arrest in the sinusoidal vascularisation of the liver. The fast-growing tumor cells create a hypoxic microenvironment which may lead to the transcriptional up-regulation of E-selectin expression in HSC. This mechanism may offer tumor cells which express sialofucosylated oligosaccharides, such as sialyl Lewis $\mathrm{x}$ (sLeX), binding sites in addition to endothelial cells. Notably, the overexpression of sLex moieties on tumor cells is correlated with poor prognosis and metastatic spread $(5,9)$.

Since we only detected a single E-selectin-binding band of $150 \mathrm{kDa}$ in HSC, it appears that HSC do not express further E-selectin binding activities such as CD44, CD43 or PSGL-1 $(7,15)$. In contrast to HSC, tumor cells often express additional P-, L- and E-selectin ligands such as CD44 and PSGL-1 (9). The up-regulation of E-selectin on activated HSC offers the potential of cell-cell interaction with endothelial and tumor cells. Notably, during the course of tissue repair after partial hepatectomy $(\mathrm{PH})$, hepatocytes and HSC first form avascular cell islands in which endothelial cells are recruited. Mice subjected to $\mathrm{PH}$ have a significantly increased number of metastases in experimental metastasis models which appears to depend on the increased E-selectin expression found after $\mathrm{PH}$ (16).

In a previous report, we demonstrated that CFR can be released into the supernatant of COS-1 cells, transiently transfected with a cDNA-endoding CFR (13). In the present study, we demonstrated that the HSC cell line LX-2 released $\mathrm{CFR}$ as an E-selectin binding protein. Hypoxia-activated HSC and MFB express less E-selectin binding activity on their cell surface than do HSC grown under normal atmospheric oxygen conditions (Figs. 2 and 4). Therefore, cleavage of the protein from the cell surface would be a potential mechanism to explain that under hypoxic conditions cell-associated CFR expression was invariant and the modifying enzyme was transcriptionally up-regulated, while less E-selectin binding activity
(ESL-1) was found on the cell surface. Since the soluble ESL-1 exported by LX-2 cells is still capable of binding to E-selectin, soluble ESL-1 may represent a competitor for cellular ESL-1.

Hypoxia-activated HSC is a new cell type expressing Eselectin and may support extravasation of the leukocyte and tumor cells into the space of Disse. It may also be speculated that HSC-derived myofibroblasts and HSC under hypoxia are capable of shedding ESL-1 from their cell surface into the extracellular space. The mode of processing and function of a soluble ESL-1, however, have yet to be elucidated.

\section{Acknowledgements}

The authors thank S. Sauer-Lehnen for excellent technical assistance. This work was supported by grants from the German Research Foundation to P.K. (KI 545/8-1) and C.H. (HE 2458/14-1 and Schn 620/3-1), and the Medical Faculty of the University of Regensburg (ReForM) to C.H.

\section{References}

1. Balabaud C, Bioulac-Sage P and Desmouliere A: The role of hepatic stellate cells in liver regeneration. J Hepatol 40: 1023-1026, 2004.

2. Friedman SL: Liver fibrosis - from bench to bedside. J Hepatol 38: S38-S53, 2003.

3. Bird NC, Mangnall D and Majeed AW: Biology of colorectal liver metastases: A review. J Surg Oncol 94: 68-80, 2006.

4. Russell J, Epstein CJ, Grisham MB, Alexander JS, Yeh KY and Granger DN: Regulation of E-selectin expression in postischemic intestinal microvasculature. Am J Physiol Gastrointest Liver Physiol 278: G878-G885, 2000.

5. Kneuer C, Ehrhardt C, Radomski MW and Bakowsky U: Selectins - potential pharmacological targets? Drug Discov Today 11: 1034-1040, 2006.

6. Zollner O and Vestweber D: The E-selectin ligand-1 is selectively activated in Chinese hamster ovary cells by the alpha $(1,3)$-fucosyltransferases IV and VII. J Biol Chem 271: 33002-33008, 1996.

7. Hidalgo A, Peired AJ, Wild MK, Vestweber D and Frenette PS: Complete identification of E-selectin ligands on neutrophils reveals distinct functions of PSGL-1, ESL-1 and CD44. Immunity 26: 477-489, 2007.

8. Willmroth F and Beaudet AL: Structure of the murine E-selectin ligand 1 (ESL-1) gene and assignment to Chromosome 8. Mamm Genome 10: 1085-1088, 1999.

9. Napier SL, Healy ZR, Schnaar RL and Konstantopoulos K: Selectin ligand expression regulates the initial vascular interactions of colon carcinoma cells: the roles of CD44v and alternative sialofucosylated selectin ligands. J Biol Chem 282: 3433-3441, 2007.

10. Shimizu S, Yamada N, Sawada T, et al: In vivo and in vitro interactions between human colon carcinoma cells and hepatic stellate cells. Jpn J Cancer Res 91: 1285-1295, 2000.

11. Antoine M, Tag CG, Wirz W, et al: Up-regulation of pleiotrophin expression in rat hepatic stellate cells by PDGF and hypoxia: implications for its role in experimental biliary liver fibrogenesis. Biochem Biophys Res Commun 337: 1153-1164, 2005.

12. Olaso E, Salado C, Egilegor E, et al: Proangiogenic role of tumor-activated hepatic stellate cells in experimental melanoma metastasis. Hepatology 37: 674-685, 2003.

13. Kohl R, Antoine M, Olwin BB, Dickson C and Kiefer P: Cysteinerich fibroblast growth factor receptor alters secretion and intracellular routing of fibroblast growth factor 3. J Biol Chem 275: 15741-15748, 2000 .

14. Dimitroff CJ, Descheny L, Trujillo N, et al: Identification of leukocyte E-selectin ligands, P-selectin glycoprotein ligand-1 and E-selectin ligand-1, on human metastatic prostate tumor cells. Cancer Res 65: 5750-5760, 2005.

15. Fuhlbrigge RC, King SL, Sackstein R and Kupper TS: CD43 is a ligand for E-selectin on CLA ${ }^{+}$human T cells. Blood 107: 1421-1426, 2006.

16. Uotani H, Yamashita I, Nagata T, Kishimoto H, Kashii Y and Tsukada K: Induction of E-selectin after partial hepatectomy promotes metastases to liver in mice. J Surg Res 96: 197-203, 2001. 